\title{
"Promesse tenue ». José Garanger et le retour au Vanuatu des objets de la sépulture de Roy Mata
}

\section{Christian Coiffier}

\section{(2) OpenEdition \\ 1 Journals}

Édition électronique

URL : http://journals.openedition.org/jso/5841

DOI : $10.4000 /$ jso. 5841

ISSN : $1760-7256$

Éditeur

Société des océanistes

Édition imprimée

Date de publication : 30 juin 2009

Pagination : 15-24

ISBN : 978-2-85430-024-6

ISSN : 0300-953x

Référence électronique

Christian Coiffier, « «Promesse tenue ». José Garanger et le retour au Vanuatu des objets de la sépulture de Roy Mata », Journal de la Société des Océanistes [En ligne], 128 | janvier-juin 2009, mis en ligne le 30 juin 2012, consulté le 01 mai 2019. URL : http://journals.openedition.org/jso/5841 ; DOI : $10.4000 / j s 0.5841$ 


\section{« Promesse tenue ». José Garanger et le retour au Vanuatu des objets de la sépulture de Roy Mata}

par

\section{Christian COIFFIER*}

\section{RÉSUMÉ}

José Garanger révolutionna les méthodes de fouilles archéologiques dans le Pacifique, mais ce fut aussi un humaniste qui avait un profond respect pour les populations vivant sur le territoire où il travaillait. Cet article vient confirmer ce fait. Il relate le processus de restitution des pièces historiques de la sépulture de Roy Mata qui eut lieu en 2000 pour répondre à une promesse faite quarante ans auparavant aux habitants du Vanuatu.

Mots-CLÉs : archéologie, ethno-histoire, Roy Mata, respect, restitution, Vanuatu

José Garanger est connu pour avoir révolutionné les recherches archéologiques dans le Pacifique en appliquant des méthodes de fouilles acquises auprès d'André Leroi-Gourhan dans la perspective d'une ethnologie préhistorique. Il eut ainsi une approche plus systématique dans les fouilles qu'il entreprit à une échelle régionale. Cet article présente un témoignage de la déontologie professionnelle de cet homme qui avait un profond respect pour les cultures locales. J'ai fait la connaissance de José à la Société des Océanistes lorsqu'il occupait les fonctions de viceprésident au sein du conseil d'administration. Mais c'est en tant que chargé des collections

\section{ABSTRACT}

José Garanger revolutionalized archaeological excavations methods in the Pacific but he was also a humanist who had the deepest respect for the populations living where he worked. This paper confirms this fact. It relates the process of restitution in 2000 of historical objects related to Roy Mata's burial ground so as to honour a promise made forty years earlier to the people of Vanuatu.

KeYwORDS: archaeology, ethno-history, Roy Mata, respect, restitution, Vanuatu

océaniennes du musée de l'Homme que je pus mesurer sa probité lorsqu'il me contacta, en 1999, par l'intermédiaire de Catherine Orliac, pour organiser le retour dans leur pays d'origine d'un ensemble de pièces archéologiques et, principalement, de celles qui provenaient de la sépulture de Roy Mata, apportées jadis en France pour étude.

Après avoir rappelé sommairement les surprenantes découvertes que l'archéologue fit dans les îles de Tongoa, Leleppa et Eretoka, à la fin des années 1960, je relaterai l'histoire du rapatriement de ces objets appartenant au patrimoine historique de la république du Vanuatu.

* Maître de conférences du MNHN et chargé de mission au musée du quai Branly, coiffier@mnhn.fr. 


\section{José Garanger et le respect des cultures locales}

José Garanger avait conscience qu'une bonne campagne de fouilles ne pouvait aboutir positivement que si l'archéologue réussissait à établir un climat de confiance avec la population vivant près du site exploré. C'est ainsi qu'il écrit :

«L'archéologue est un intrus dans la société autochtone. Les simples relations d'employeur à employés, sanctionnées par un échange monétaire, sont ici insuffisantes. Croyances et traditions veillent sous les attitudes modernisées et il y a grande indiscrétion à soulever ce voile et plus grande indiscrétion encore à toucher au domaine des morts. Il est donc non seulement nécessaire de se faire accepter mais encore indispensable d'obtenir que sa curiosité ne soit pas indiscrète et que le groupe lui-même participe à l'intérêt de sa recherche. » (Garanger, 1972 : 18)

José Garanger transmit cette déontologie de son métier à deux générations de jeunes archéologues. La méthode d'approche du terrain et l'organisation de la fouille en coopération avec les autorités locales était pour lui la clé de la réussite. L'ethnologue Jean Guiart (1996: 40) reconnait que la démarche du professeur Garanger a été l'un de ses apports méthodologiques importants. En Océanie, toute intervention dans le domaine des morts interfère avec celui des vivants car il n'y a pas de réelle barrière entre ces deux mondes comme en Occident. José Garanger était un homme pragmatique qui savait écouter les uns et les autres pour tirer de leurs discours les précieux indices qui allaient donner un sens à ses recherches. C'est en confrontant ainsi la tradition orale mythologique à la réalité scientifique qu'il put comprendre que le passé historique local avait été masqué par les nouvelles idéologies apportées par les Européens depuis deux cents ans. Il en tira la conclusion suivante :

«Aucune civilisation ni aucun homme ne peut vivre sans passé. Le malaise est grand dans ces îles mélanésiennes où le passé des hommes fut trop brutalement voué aux enfers, où le présent n'est qu'une incessante hésitation entre le désir de s'opposer et l'effort de s'adapter à des pensées religieuses, à des principes d'autorité, à des systèmes socio-économiques étrangers. » (Garanger, 1972 : 15)

Sa méthodologie, associant plusieurs sciences comme l'ethnologie, l'histoire et l'archéologie, se révéla très constructive dans le développement des recherches interdisciplinaires en sciences humaines dans les archipels du Pacifique. Elle permit de développer les connaissances générales sur le passé de l'Océanie et particulièrement sur celui du Vanuatu. En mettant au jour les restes d'un passé qui n'était connu que par la tradition orale, José Garanger a fait ressurgir la fierté des ni-Vanuatu vis-à-vis de leur histoire.

\section{Les recherches archéologiques de José Garanger au Vanuatu}

Les recherches archéologiques de José Garanger aux Nouvelles-Hébrides (l'actuel Vanuatu) se firent dans le cadre d'une mission conjointe franco-américaine (1972: 9) placée sous l'égide de l'orstom (l'actuel IRD) pour le côté français. Les îles du sud et celles du nord furent fouillées par les archéologues américains Mary Elisabeth et Richard Shutler. De son côté, José Garanger fut chargé en 1964 et en 1966-1967 d'effectuer des fouilles dans les îles du centre du Vanuatu (nord d'Efate, Makura et Tongoa) pour étudier les niveaux pré-et post-volcaniques et tenter de vérifier les données de la tradition concernant l'ancienne Kuwae. Dès 1964, José Garanger s'enquit de savoir où se trouvait Mangaasi, le lieu du farea de Roy Mata, le chef principal de l'île d'Eretoka évoqué par les informateurs de l'ethnologue Jean Guiart. Après avoir terminé ses travaux à Leleppa, en 1967, il revint à Mangaasi pour prospecter et faire le relevé général de ce site. L'endroit présumé de la sépulture de Roy Mata était considéré comme tabou. Il fallut toute l'habileté et le professionnalisme de José Garanger pour obtenir des autorisations de fouilles (Garanger, 1996 : 73). En tout, près d'une quarantaine de squelettes fut découverte dans cette sépulture collective. Ces derniers ne furent pas déplacés, mais seulement photographiés et étudiés sur place.

José Garanger ne prélèva que des parures (bracelets en dent de cochon arrondie, colliers et pendentifs taillés dans des dents de cétacés, brassards en perles, bijoux de coquillages) associées à la position sociale des défunts. Ce sont ainsi plusieurs centaines de tessons de poteries mais également des éléments d'outils lithiques, de corail ou de coquillages, trouvés lors des précédentes fouilles dans les îles de Tongoa et d'Efate qui furent apportés en France pour y être étudiés et analysés (Garanger, 1972: 59). Avant son départ des Nouvelles-Hébrides, José Garanger fit la promesse aux chefs coutumiers et aux autorités administratives que cet ensemble serait restitué ultérieurement, en l'occurrence dès que toutes les études auraient été terminées et publiées. Ces matériaux lui permirent de présenter à la Sorbonne une thèse de doctorat es-lettres dont l'essentiel fut publié par la Société des Océanistes et l'orstom sous le titre Archéologie des Nouvelles-Hébrides, contribution à la connais- 
sance des îles du Centre (1972). En 1980, José Garanger, sous son propre nom, mit en dépôt, au département d'Océanie du musée de l'Homme soixante et une pièces sous le numéro de collection M.H. 980 D.113.

\section{Préparation du rapatriement des pièces archéologiques}

En 1980, le condominium des NouvellesHébrides accéda à l'indépendance sous le nom de République du Vanuatu. Paul Gardissat, un des membres fondateurs de Radio-Vanuatu, demanda au comité d'organisation des fêtes de l'indépendance d'inviter José Garanger qui accepta volontiers et réalisa à cette occasion avec celui-ci et Kirk Huffman (directeur du Centre culturel) un enregistrement radiophonique de plus d'une heure sur la découverte de la sépulture de Roy Mata. Paul Gardissat réalisa par ailleurs de nombreux enregistrements sur la tradition orale de Roy Mata ${ }^{1}$. José Garanger souhaita restituer au nouvel État l'ensemble de ses découvertes archéologiques et particulièrement celle d'Eretoka. Il avait conscience de l'importance historique de cette sépulture multiple, mais il lui semblait primordial d'attendre que des conditions de sécurité et de conservation convenables fussent réunies à Port-Vila. Il avait conscience en effet que la pérennité de ces objets avait une importance primordiale pour le patrimoine culturel de la jeune République. Durant près de vingt ans, José Garanger œuvra, avec ses amis ni-Vanuatu, à valoriser l'histoire de leur pays à partir de ses découvertes. Dès 1992, il était entré en contact avec les autorités compétentes du Vanuatu pour organiser le retour des collections emportées en France. Quelques années plus tard, ce projet prit corps sous l'impulsion de Catherine Orliac, responsable du GDR 1170 du CNRS avec l'aide d'un étudiant en archéologie, Marcellin Abong, originaire de l'île de Malakula. Ce boursier de l'ambassade de France à Port-Vila poursuivait des études à l'Institut d'art et d'archéologie tout en suivant une formation en xylologie à Paris vi sous la direction de Catherine Orliac. C'est avec l'aide de Jean-Christophe Galipaud qu'elle commença alors un travail de classement et la réalisation d'inventaires à
Noisy-le-Grand. À plusieurs reprises, José Garanger tenta de rapporter lui-même au Vanuatu quelques pièces provenant de ses fouilles, mais il n'existait pas à cette époque d'institution spécialisée capable de les conserver dans de bonnes conditions, si bien que certaines furent détruites lors d'un cyclone et que d'autres se trouvèrent gravement endommagées par la pressurisation régnant à l'intérieur des avions lors des vols aériens entre Paris et Port-Vila. La construction du nouveau Centre culturel du Vanuatu à Port-Vila en 1995 allait fournir l'opportunité de lancer le projet d'une grande exposition internationale sur le Vanuatu. Il participa à l'élaboration de ce projet avec le nouveau directeur du Centre, Ralph Regenvanu, originaire de l'île d'Uripiv et jeune diplômé de l'Université nationale d'Australie, Joël Bonnemaison, directeur de recherche à l'oRstom, JeanChristophe Galipaud et David Roe, co-responsables du projet d'inventaire des sites historiques et culturels au sein du ministère de la culture du Vanuatu (vchss). Marcellin Abong fut chargé du suivi du dossier Roy Mata. Le nouvel établissement était alors à même d'exposer des objets et d'en recevoir en prêt. José Garanger travailla activement avec les commissaires de cette exposition, Christian Kaufmann, Roger Boulay et Kirk Huffman pour mettre au point à destination d'un public local une présentation d'un ensemble de pièces provenant de ses fouilles. L'exposition internationale Vanuatu. Océanie. Arts des iles de cendre et de corail s'ouvrit à Port-Vila le 28 juin 1996 et elle fut ensuite présentée à Nouméa, Bâle et Paris. C'est José Garanger qui rédigea la préface du catalogue, ainsi qu'un article destiné principalement aux lecteurs locaux. Il y présenta ses découvertes de Tongoa ainsi que celles de Mangaasi et d'Eretoka (Garanger, $1996: 8-11,68-75)$. Il veilla à ce qu'une version en bislamar soit publiée pour que le plus grand nombre puisse comprendre le but de ses recherches. L'exposition eut un très grand succès, particulièrement au Vanuatu où cet événement, intitulé, Spirit blong bubu i kam bak («Le retour des esprits des ancêtres") acquit une portée nationale (Conseil national culturel de Vanuatu, 1996) ${ }^{2}$. José Garanger souhaita que les résultats de ses fouilles pussent servir à l'édu-

1. Tous ces documents sonores furent versés, quelques années plus tard, au dossier de demande de classement du site de Roy Mata au patrimoine mondial de l'humanité.

2. Le petit guide en bislamar évoque, à la page 9, des objets exposés dans l'exposition : «- Sam samting we wan arkiolojis blong Franis José Garanger i bin faenem long grev blong Roimata long Retoka (Hat Aelan), we Paul Gardissat i bin mekem wan eksibisen long olgeta long Kaljarol Senta long taem blong Indipendens long 1980. » («Quelques objets qu'un archéologue français, José Garanger, a découvert dans la tombe de Roy Mata à Eretoka - l'île chapeau -, exposés par Paul Gardissat lors de l'exposition au Centre culturel à l'occasion de l'Indépendance en 1980. »). 


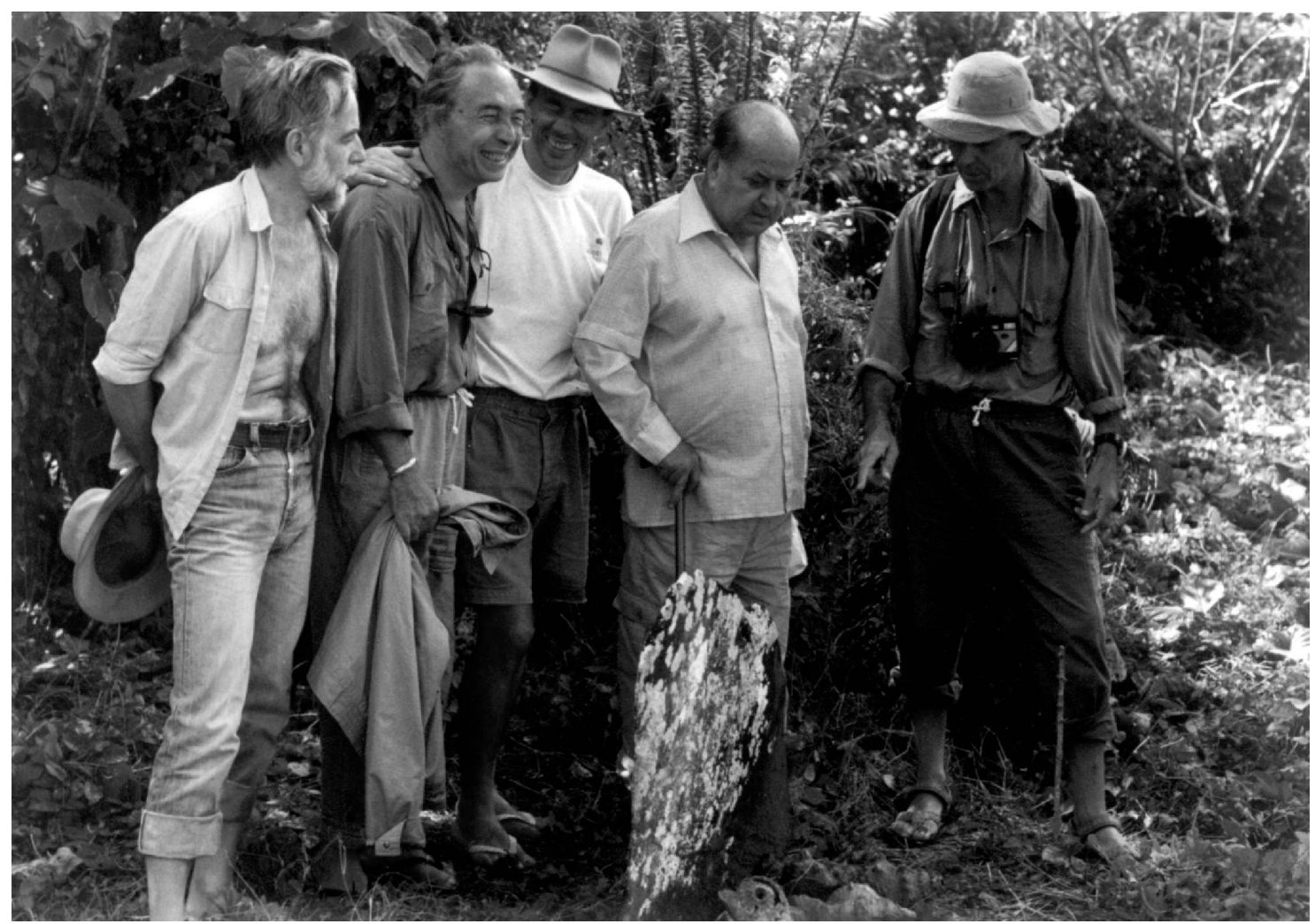

Рното 1. - José Garanger entouré de gauche à droite de Jean-Pierre Siorat, Daniel Frimigaci, Jean-Christophe Galipaud et Jean-Michel Chazine devant la tombe de Roy Mata (avec l'aimable autorisation de J.-P. Siorat)

cation de la jeunesse locale. Il se rendit plusieurs fois au Centre culturel pour s'occuper de la présentation pédagogique des pièces archéologiques qu'il avait trouvées. En 1996, il participa au colloque Lapita à Port-Vila, ce qui lui donna l'occasion de retourner sur ses terrains de fouilles (photo 1). Il profita de cet événement pour restituer officiellement des éléments de parure du site d'Eretoka. Ceux-ci furent exposés dans la galerie supérieure du musée au centre culturel de Port-Vila.

Dans cette nouvelle conjoncture, José Garanger put enfin envisager sérieusement de réaliser sa promesse de restitution à leur pays d'origine des pièces collectées dans les années 1960. Comme il ne se sentait plus la force de se déplacer jusqu'au Vanuatu pour effectuer lui-même cette restitution, il me contacta et demanda à ses collaborateurs de l'aider. Il se trouvait qu'il conservait dans sa demeure de Noisy-le-Grand diverses pièces archéologiques qu'il y avait amenées par souci de sécurité car le laboratoire dont il était le directeur avait déménagé à plusieurs reprises. Dans le courant de l'année 1998, plusieurs personnes se mobilisèrent. Catherine Orliac, aidée de Marcellin Abong, se chargea de rassembler et de transporter au département d'Océanie du musée de l'Homme des caisses de tessons de poterie, d'outils lithiques ou en coquillage, des parures et des monnaies.

Le directeur du Centre culturel, Ralph Regenvanu, entreprit alors des démarches officielles auprès des autorités françaises. Il me fit transmettre une lettre au musée de l'Homme par l'intermédiaire de Jean-Georges Mandon, attaché culturel à l'ambassade de France au Vanuatu. Dans celle-ci, il réclamait une aide financière pour le rapatriement de ces objets en argumentant que ceux-ci pourraient ensuite êtres utilisés avec dans un but éducatif et pour des recherches ultérieures dans le pays. Il fit également remarquer que le musée national du Vanuatu était maintenant prêt à recevoir, stocker et exposer ces matériaux, tout particulièrement s'ils avaient bénéficié au préalable du traitement de restauration requis en France, avant leur rapatriement. Il conclut sa lettre par ces mots :

« Je suis convaincu que vous estimerez à sa juste valeur ce projet de restituer une part importante de l'héritage du Vanuatu au profit du pays et de ses citoyens. En outre, étant donné que la recherche du professeur Garanger au Vanuatu fut entreprise sous les auspices du gouvernement français, il semble approprié que le gouvernement français garantisse également la restitution de ces matériaux au peuple du 


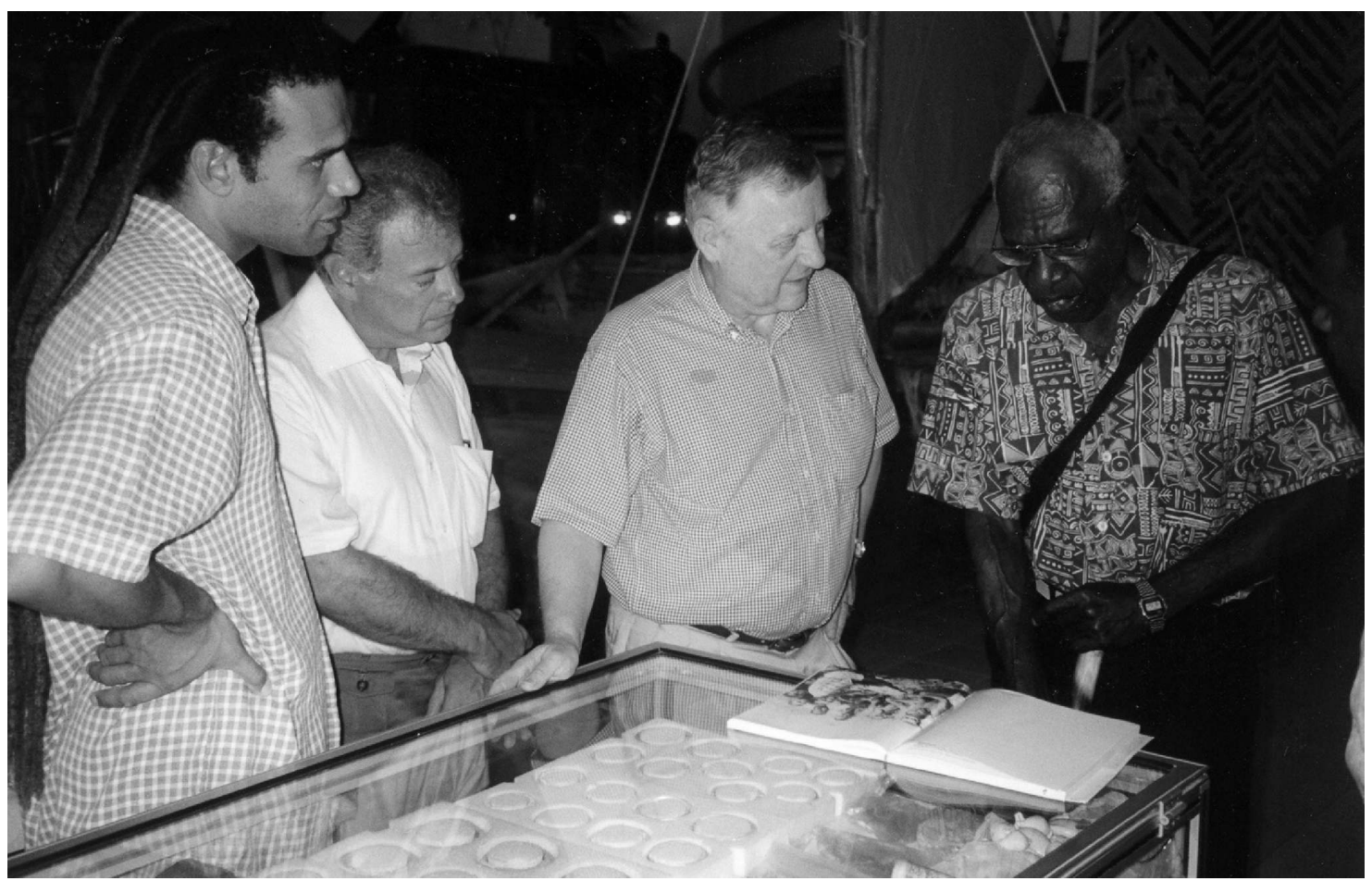

Рното 2. - Ralph Regenvanu, Jean-Claude Moreno, Patrick Amiot (ambassadeur de France au Vanuatu) et Kaltaua Munalapa, un ancien membre de l'équipe de fouille d'Eretoka devant les parures restituées et la photo de l'équipe dans les années 1960 publiée dans l'ouvrage de Garanger (1972 : 159) (cliché de C. Coiffier)

Vanuatu, dans notre Musée national. » (lettre à JeanGeorges Mandon, 3 mai 1999, dossier José Garanger, archives du MQB).

Un inventaire complet de tous les objets provenant des collections Garanger déposées au musée de l'Homme fut confié à une collègue de Catherine Orliac, Suzanne Vinette, en novembre 1999. Le restaurateur Alain Renard, consulté au sujet des conditions de transport jusqu'au Vanuatu, estima que certaines pièces devaient être restaurées pour pouvoir supporter les changements climatiques. Une année de négociations au Vanuatu et à Paris fut nécessaire pour arriver à organiser le rapatriement des collections de José Garanger ( $c f$. annexes). À la fin de l'été 2000, Jean-Claude Moreno, en sa qualité d'administrateur provisoire du Muséum national d'histoire naturelle, fit part de sa décision de venir remettre officiellement ces collections au mois d'octobre de la même année au Centre culturel du Vanuatu (lettres à Ralph Regenvanu datées des 18 août et 27 septembre 2000, archives du MQB).

\section{Promesse tenue}

Le choix de restituer l'ensemble des collections Garanger lors d'une même manifestation fut donc accepté par toutes les parties et une date fut fixée au début du mois de novembre 2000. Marie-Claire Bataille, du musée de l'Homme, accepta de suivre la phase d'emballage et de déménagement. La secrétaire de Jean-Claude Moreno se vit confier la responsabilité d'accompagner et d'acheminer à bon port ces collections vers Port-Vila, via Nouméa. Les objets les plus précieux et les plus fragiles furent alors placés dans une valise métallique hermétique et le reste dans des caissons en bois placés dans trois grandes caisses. José Garanger avait convenu, avec l'accord des scientifiques du Vanuatu, qu'un certain nombre de pièces seraient conservées en France pour répartir les risques d'une éventuelle destruction. Pierre Ottino et Catherine Orliac furent chargés de faire une sélection de vingtdeux pièces qui seraient données au musée de l'Homme (voir le dossier de restitution établi le 30 octobre 2000 par Marie-Claire Bataille et conservé dans les archives du MQB). Il s'agissait d'objets ou de parures qui n'étaient pas en excellent état et qui constituaient des doublons dans la collection.

Le 31 octobre, Michèle Nespoulet et moimême arrivâmes avec les collections à Port-Vila via Nouméa. Le constat d'état effectué avec le personnel du musée ne mit en évidence aucun dégât qui aurait pu être survenu durant le transport. La cérémonie officielle de remise des collec- 


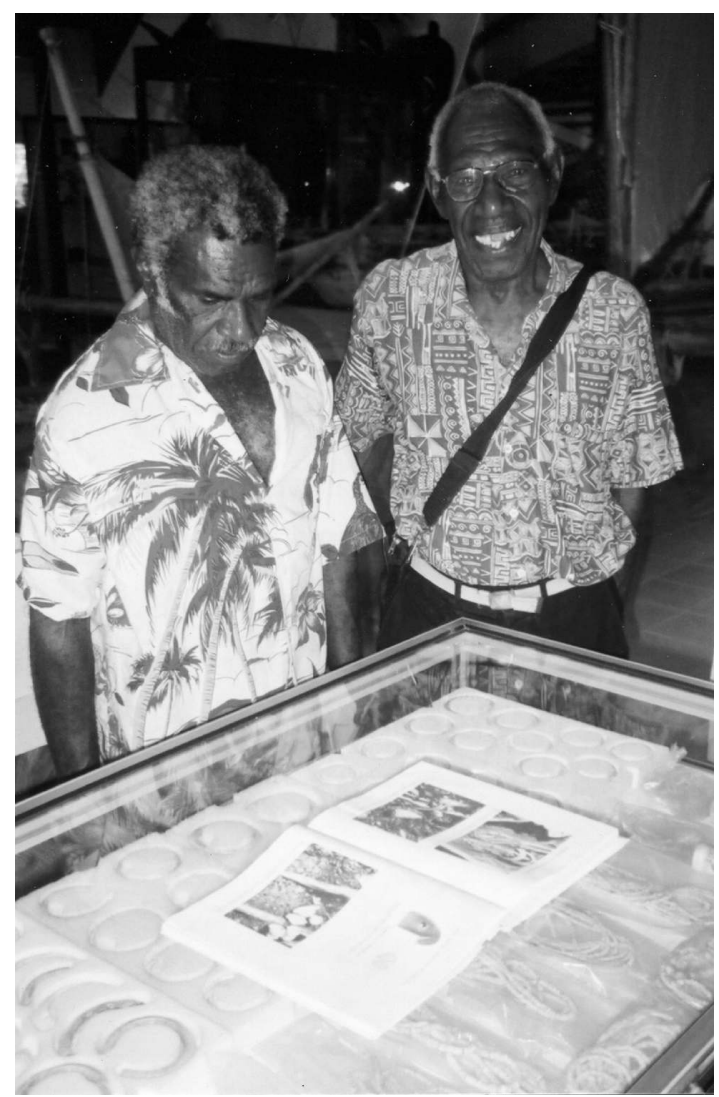

Рното 3. - Langas Meto Kalotiti et Kaltaua Munalapa, deux anciens membres de l'équipe de fouilles dirigée par José Garanger à Eretoka devant une vitrine présentant quelques parures de la sépulture de Roy Mata (cliché de C. Coiffier)

tions Garanger, comprenant les objets historiques de Roy Mata, eut lieu trois jours plus tard au Centre culturel du Vanuatu en présence de nombreuses personnalités officielles (photos 2 et 3).

La presse du Vanuatu se fit l'écho du retour de ces pièces historiques avec un article intitulé «Roimata's jewellery artifacts come home» (Trading Post, 11 novembre 2000, archives du $\mathrm{MQB})$ et accompagné d'une photo de Marcellin Abong et Takaronga posant devant les parures de Roy Mata. En Nouvelle-Calédonie comme au Vanuatu, la radio consacra plusieurs émissions à l'événement. Ralph Regenvanu exprima alors toute sa gratitude et ses remerciements à José Garanger en écrivant une lettre en ces termes :

« Même si vous n'êtes pas présent lors de la cérémonie de la remise officielle des objets au Centre Culturel nous pensons bien à vous sincèrement. Vous aviez souhaité depuis toujours le retour de ces objets au pays et votre souhait est devenu concret en ce jour du 4 novembre 2000. » (Lettre au professeur José Garanger, 9 novembre 2000, archives du MQB)

Quelques semaines après la cérémonie de Port-Vila, Ralph Regenvanu fut accueilli à Paris.
Il délivra, le jeudi 14 décembre, une conférence au musée de l'Homme, organisée par la Société des Océanistes, sur le retour au Vanuatu de ce matériel archéologique. Il se rendit au domicile de José Garanger à Noisy-le-Grand pour lui faire le compte-rendu des derniers événements de Port-Vila et lui en présenter les photos. Celui-ci se montra très ému et satisfait de constater que sa promesse avait été tenue (photos 4 et 5).

En tant que responsable du dossier Roy Mata, Marcellin Abong ne manqua pas de remercier à son tour par courrier toute l'équipe du muséum qui avait participé au succès de l'opération (lettre de Marcellin Abong à Christian Coiffier, 14 novembre 2000, archives du MQB).

Un ensemble de pièces provenant des fouilles de José Garanger est désormais présenté dans une vitrine du musée du Centre culturel à PortVila avec des textes évoquant l'épopée de Roy Mata. Les écoliers du Vanuatu peuvent ainsi visualiser les traces matérielles d'un grand chef qui tient une place reconnue dans l'histoire de leur pays.

\section{Conclusion}

José Garanger a su utiliser judicieusement les données historiques présentes dans les mythes locaux pour y trouver les bases de ses recherches archéologiques. C'est en diffusant cette méthode qu'il forma deux générations de chercheurs océanistes qui poursuivent ses travaux avec la même déontologie. Dans sa thèse, l'archéologue présente les résultats de ses recherches comme une extraordinaire avancée dans la reconnaissance par les ni-Vanuatu de leurs structures sociales et de leur histoire commune :

«La redécouverte de ce passé par les recherches archéologiques pourrait être plus fructueuse que les expériences irrationnelles des mouvements de libération par le renouvellement et la transformation des mythes. Ce n'est pas là simple spéculation de l'esprit, les résultats acquis au cours de ces deux missions archéologiques n'auraient pu l'être sans le concours des Autochtones. Une fois qu'il leur fut démontré que les recherches préhistoriques pouvaient aider à confirmer et à préciser la véracité de certaines de leurs traditions, ils devinrent eux-mêmes des assistants très attentifs et actifs, et à ce point intéressés que plusieurs sites ont été étudiés parce qu'ils le demandaient. Puisse ce premier essai les aider quelque peu dans la quête de leur passé, puissent, certains d'entre eux, découvrir et écrire eux-mêmes, bientôt, leur propre histoire ! » (Garanger, 1972 : 16)

Les souhaits de José Garanger, pour la préservation du patrimoine qu'il a contribué à mettre au jour, se sont finalement réalisés puisque plu- 


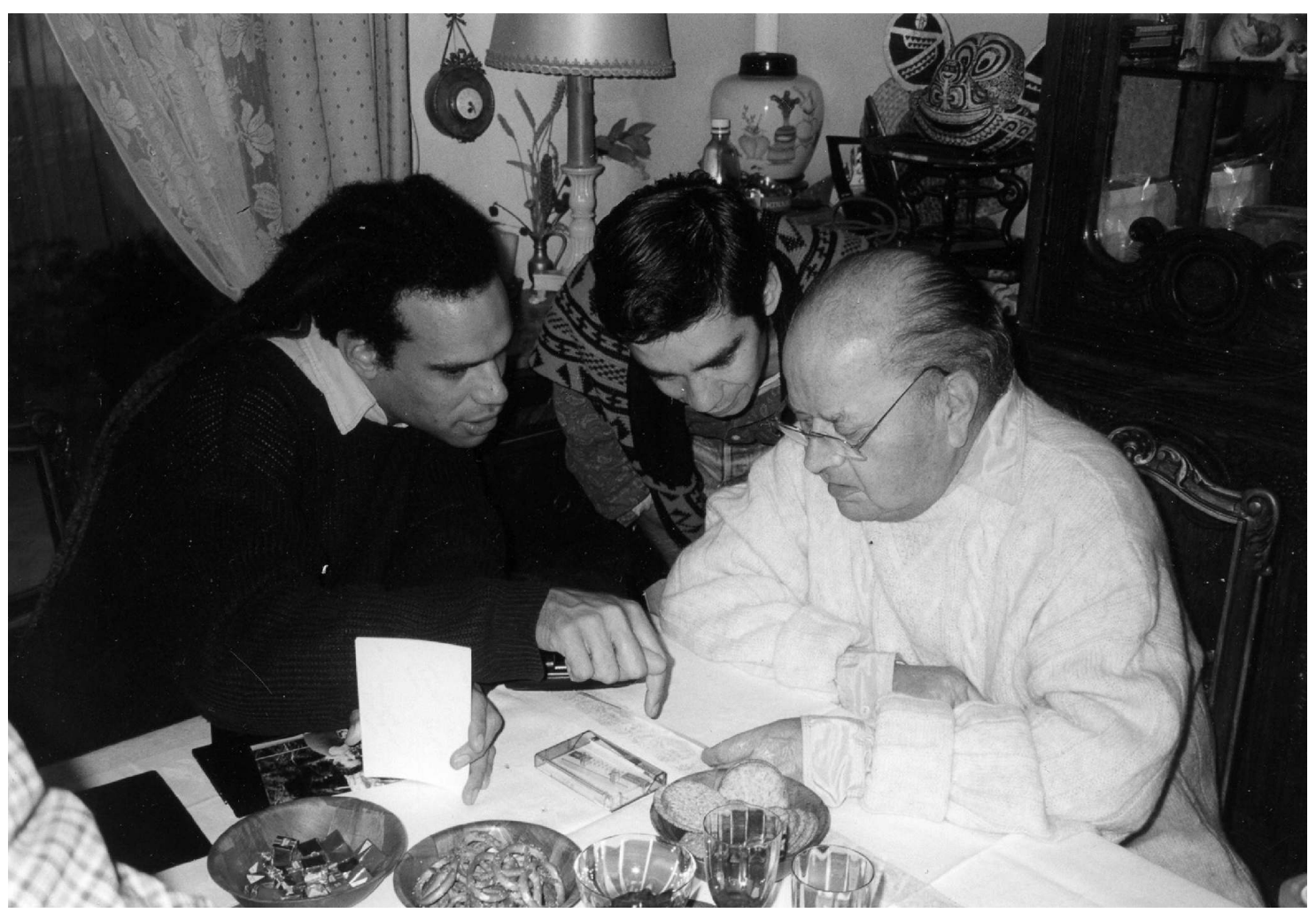

Рното 4. - À Noisy-le-Grand, Ralph Regenvanu (à gauche) présente à José Garanger (à droite) et à Pierre Ottino (au centre) des photos de la cérémonie de restitution à Port-Vila (cliché de C. Coiffier)

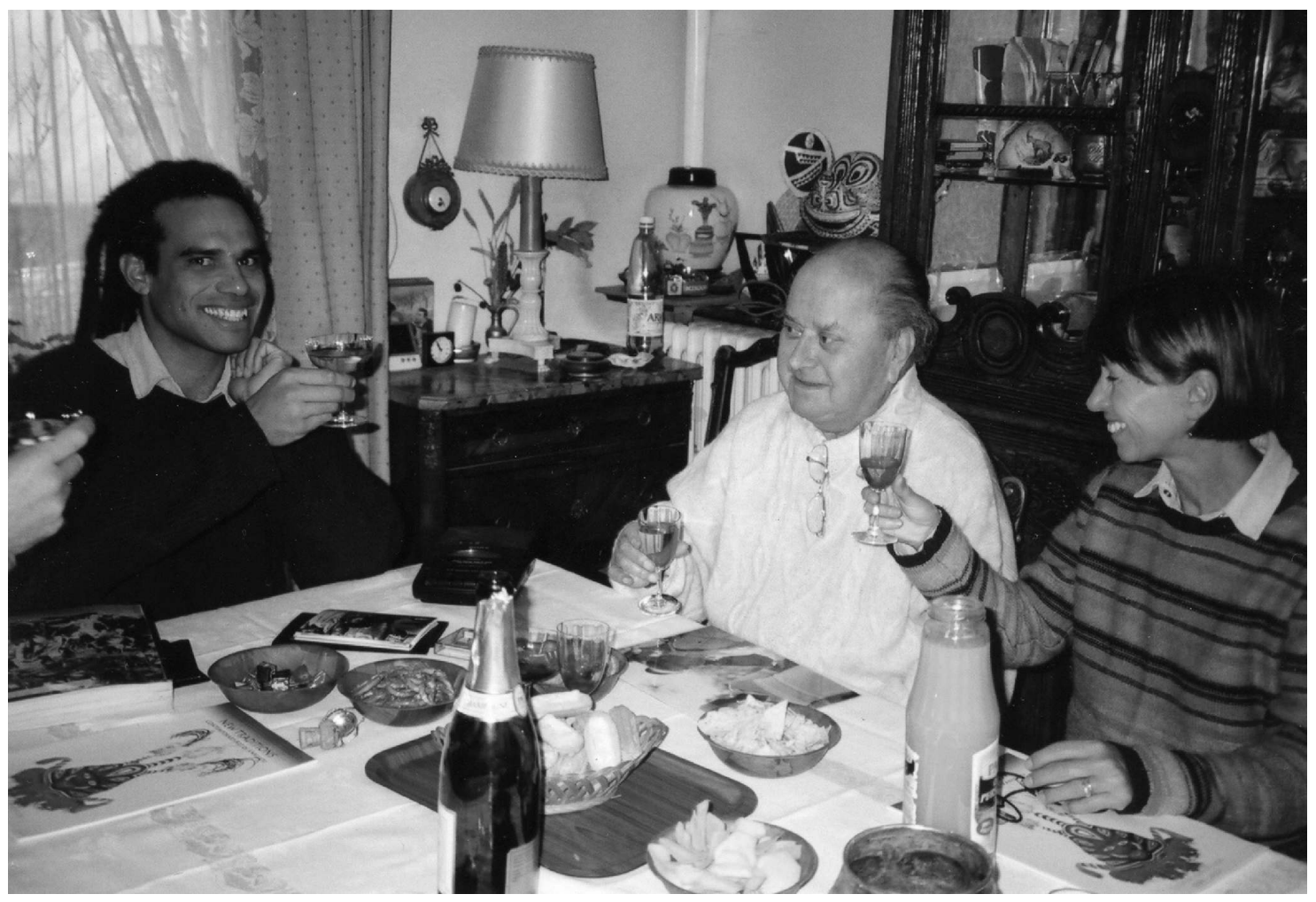

Рното 5. - À Noisy-le-Grand, Ralph Regenvanu (à gauche) et Catherine Orliac (à droite) trinquent avec José Garanger (au centre) à la promesse tenue (cliché de C. Coiffier) 
sieurs ni-Vanuatu sont aujourd'hui diplômés en archéologie et Marcellin Abong est devenu directeur du Centre culturel de Port-Vila. Je conclurai cet hommage en méditant cette pensée de José Garanger pour qui la relation avec ses collaborateurs ni-Vanuatu était primordiale :

«J'ai découvert une valeur humaine insoupçonnée et exceptionnelle, une amitié profondément partagée. Cette pudique compréhension mutuelle est bien le plus important résultat de cette quête scientifique du passé. » (1996 : 75)

\section{Remerciements}

L'auteur remercie Catherine Orliac, Paul Gardissat, Christian Kaufmann et Jean-Christophe Galipaud pour les informations qu'ils lui ont transmises concernant l'histoire de ce rapatriement (avant 1999).

\section{BIBLIOGRAPHIE}

Conseil national Culturel de Vanuatu, 1996. Spirit Blong Bubu I Kam Bak, Arts of Vanuatu, Arts de Vanuatu, 29 june-10 august 1996, Port Vila, Kaljoral Senta blong Vanuatu.

Garanger José, 1972. Archéologie des NouvellesHébrides, contribution à la connaissance des îles du Centre, Paris, Société des Océanistes (avec l'ORsTOM), Publications de la Société des Océanistes 30.

_, 1996. Introduction, in J. Bonnemaison, K. Huffman, Ch. Kaufmann et D. Tryon (éds), Vanuatu Océanie. Arts des îles de cendre et de corail, Paris, Éditions de la Réunion des musées nationaux, pp. 8-11.

-, 1996. Tongoa, Mangaasi et Eretoka, histoires d'une préhistoire, in J. Bonnemaison, K. Huffman, Ch. Kaufmann et D. Tryon (éds), Vanuatu Océanie. Arts des îles de cendre et de corail, Paris, Éditions de la Réunion des musées nationaux, pp. 68-75.

Guiart Jean, 1996. Archéologie et ethnologie, in Michèle Julien, Michel Orliac et Catherine Orliac (éds), Mémoire de pierre, mémoire d'homme, tradition et archéologie en Océanie. Hommage à José Garanger, Paris, Publications de la Sorbonne, Homme et Société 23, pp. 31-63.

\section{ANNEXE}

\section{Chronologie des différentes démarches (dossier José} Garanger, musée du quai Branly)

1. L'archéologue Jean-Christophe Galipaud convainc en 1999 des responsables de son institution,
l'IRD, d'aider financièrement le projet et se propose pour acheminer les collections jusqu'au Vanuatu lors de l'une de ses prochaines missions.

2. Les crédits nécessaires pour la restauration et pour le transport de la seconde collection sont réunis suite à un échange de correspondance entre Catherine Orliac, Jean-Christophe Galipaud, Michel Lardy (représentant de l'IRD à Port-Vila) et Madame Voisin (Maison de l'Archéologie et de l'Ethnologie de Nanterre) (courriers électroniques des 26 août, 25 octobre, 3, 4 et 17 novembre, 17 décembre 1999, archives du $\mathrm{MQB})$.

3. De nombreux problèmes restent en suspens par rapport à la sortie légale des collections du territoire français alors que l'engagement budgétaire doit se faire avant le 30 novembre 1999.

4. À la fin de l'année 1999, la restauration des objets de la première collection n'est toujours pas terminée, faute de financement et de coordination générale des diverses actions.

5. L'ambassade de France au Vanuatu annule les crédits alloués en raison d'une confusion entre les travaux de restauration payés par l'IRD pour les objets conservés au musée de l'Homme et ceux qui étaient envisagés pour la collection provenant de chez le professeur Garanger.

6. Catherine Orliac rédige une lettre à la secrétaire générale de l'ambassade de France à Port Vila, Madame Madraux (21 décembre 1999, archives du MQB).

7. Ralph Regenvanu confirme que le Musée national du Vanuatu est prêt à recevoir les collections Garanger (lettre à Christian Coiffier du 22 novembre 1999, archives du MQB).

8. Un nouveau problème administratif surgit car la résiliation du dépôt au musée de l'Homme ne peut émaner que d'une demande officielle de l'ayant droit, en l'occurrence José Garanger lui-même. Ce dernier adresse une lettre au directeur du laboratoire d'ethnologie pour lui faire part officiellement de son désir de récupérer sa collection (lettres à Pierre Robbe de novembre 1999 et du 29 juin 2000, archives du MQB).

9. En avril 2000, Ralph Regenvanu reçoit confirmation de l'ambassade de France du fait que le rapatriement de la collection Garanger à Port-Vila donnera lieu à une cérémonie spéciale, mais il n'y a toujours pas de ligne de crédit pour restaurer les objets (lettre de Jean-Georges Mandon à Ralph Regenvanu du 14 avril 2000, archives du MQB).

10. Les différentes démarches déjà entreprises sont récapitulées dans une lettre adressée au directeur du Centre culturel du Vanuatu (lettre à Ralph Regenvanu du 20 juillet 2000, réf. 790, archives du MQB) avec copies à Madame Madraux (secrétaire générale de l'ambassade de France à Port Vila), Jean-Claude Moreno (administrateur provisoire du Muséum national d'histoire naturelle), Catherine Orliac et José Garanger (lettres à Madame Madraux du 20 juillet 2000, réf. 792, 791, 794 \& 793, archives du MQB).

11. La situation se débloque au cours de l'été 2000 grâce à l'intervention de Jean-Claude Moreno, qui confirme que le Muséum accepte de prendre en charge la restauration du second lot d'objets et les frais de 
transport des deux collections. Il demande à sa secrétaire, Michèle Nespoulet, de s'occuper du dossier (lettre de C. Coiffier à J.-C. Moreno du 9 août 2000, archives du MQB).

12. Les dix-sept objets restant à restaurer sont confiés au restaurateur Alain Renard qui réalise des emballages adaptés pour chacune des pièces (septembre 2000).
13. Ralph Regenvanu adresse ses remerciements à toutes les personnes ayant œuvré pour mener ce projet à son terme (lettre à C. Coiffier du 26 septembre 2000, archives du MQB).

14. Discours de Jean-Claude Moreno au Centre culturel lors de la cérémonie officielle du 4 novembre 2000 (archives du MQB). 
